\title{
Synthesis of mono- and diboronic acid calix[4]arene receptors
}

\author{
Jeremy D. Glennon*, Andrew Walker, and M. Anthony McKervey \\ Analytical and Biological Chemistry Research Facility, Department of Chemistry, \\ University College Cork, Cork, Ireland \\ E-mail: j.glennon@ucc.ie
}

Dedicated to our co-author Professor M. Anthony McKervey on his $65^{\text {th }}$ birthday

(received 21 Mar 03; accepted 18 Jul 03; published on the web 19 Jul 03)

\begin{abstract}
Various boronic acid appended calixarene assemblies were constructed on the macrocyclic lower rim. Treatment of the appropriate calix[4]arene with controlled amounts of base and 2bromomethylphenylethylene boronate affords the monoboronic acid triphenol or the distally separated diboronic acid of choice. Upper rim $p$-allyl calix[4]arene phenyl boronic acids were prepared. These ligands were designed to allow further study of guest complexation in nonaqueous, aqueous and on solid substrates.
\end{abstract}

Keywords: Calixarene, boronic acid

\section{Introduction}

There is considerable evidence of the interest in the Lewis acid binding properties of organoboron compounds for organic synthesis and molecular recognition. ${ }^{1}$ Trigonal $\mathrm{sp}^{2}$ hybridized boronic acids $\mathrm{RB}(\mathrm{OH})_{2}$ bind hydrophilic diols, either by the reversible formation of a neutral trigonal boronate ester, or through a mechanism which is considered to be more favourable involving an ion-paired $\mathrm{sp}^{3}$ hybridized tetrahedral anion. In addition, complex formation between boronic acids and polyols ${ }^{2}$ forms the basis of chromatographic separations and solid phase extraction chemistries; for example, Hagemeier et al. have utilized silica-bonded $m$-aminophenylboronic acid for the chromatographic analysis and elution (under acidic conditions) of cis-diols containing ribonucleosides and ribonucleic acids in clinical samples. ${ }^{3}$ Several boron carrier molecules have been prepared for anti-cancer treatment by boron capture therapy, including a phenylboronic acid porphyrin derivative. ${ }^{4}$

There is a wealth of literature in chemical journals and in specialised books concerning the

synthesis and properties of calixarenes. ${ }^{5}$ Our laboratory has been active in designing and synthesizing calixarenes for selective metal extraction and for use as chromatographic stationary 
phases. Silica bonded calixarene phases have been prepared, characterized by solid state CP MAS NMR ${ }^{6}$ and shown to be selective for alkali metals, when studied as stationary phases in water eluent ion chromatography. ${ }^{7}$ The first examples of the use of calixarenes in supercritical fluids have been reported by this laboratory. ${ }^{8}$ The fluorination of calixarenes at the upper rim provides a convenient means to increase the solubility of these 'molecular baskets' in sc- $\mathrm{CO}_{2}$, while lower rim functionalisation presents the opportunity for location of suitable donor atoms for selective complexation. ${ }^{9}$

The synthesis of fluorescent boronic acid calixarenes through functionalization at the opposite upper rim, has been reported by Shinkai and co-workers. ${ }^{10}$ No work on lower rim functionalized boronic acid calixarenes with the design proposed here has appeared in the literature.

\section{Results and Discussion}

The incorporation of a phenylboronic acid unit to a crown ether substructure has been described by Tuladhar and D'Silva using 2-bromo-1,3-xylene ${ }^{11}$ and a similar approach is used here for the attachment of phenylboronic acid podands to calixarene templates. By using o-bromotoluene, a single arm fixation to the lower rim of the calixarene is possible. Exposure of tri- $n$-butyl borate ${ }^{12}$ to the Grignard reagent from o-bromotoluene $\mathbf{2}$ in dry THF at $-70^{\circ} \mathrm{C}$ afforded the boronic acid $\mathbf{4}$, in moderate yield ${ }^{13}$ after hydrolysis as depicted in Figure 1. Free radical bromination of ethylene glycol protected 2-methylphenylboronic acid $\mathbf{5}$ afforded the $\alpha$-bromo product $\mathbf{6}$ which can be used to attach such boronate ligands to the host calixarene architecture.<smiles>Cc1ccccc1Br</smiles>

1<smiles>Cc1ccccc1B(O)O</smiles>

4<smiles>Cc1ccccc1B1OCCO1</smiles>

5
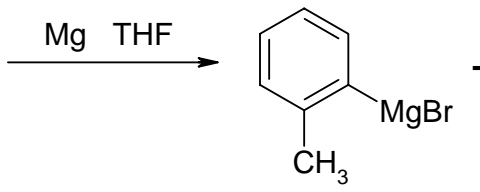

2

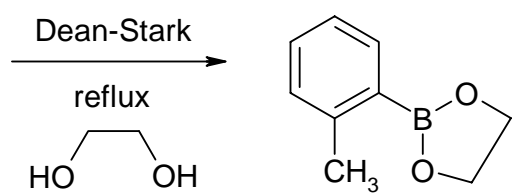

5

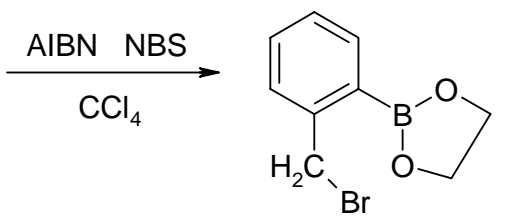

6

Figure 1 


\section{Route to Mono- and Disubstituted Calix[4]arene boronic acids}

The synthetic methodology for the monosubstitution of calix[4]arenes was first devised by Reinhouldt and co-workers ${ }^{14}$ and was adapted for the monoboronation of $p$-tert-butyl-, $p$-allyl and $p$-Hcalix[4]arenes.

Treatment of the appropriate calix[4]arene with limiting amounts of base and 2bromomethylphenylethylene boronate $\mathbf{6}$ affords the crude monoboronic acid calixarene as shown in Figure 2. Increasing the proportion of base allows the incorporation of distally separated diboronate functionalities $\mathbf{9}$. Purification by chromatography eluting with dichloromethane:ethyl acetate $(10: 1)$ was necessary in each case to isolate the desired mono- and diboronic acids products.<smiles>CC(C)(C)Cc1cc(C(C)(C)C)cc(C(C)(C)C)c1O</smiles>

7<smiles>CC(C)(C)Cc1cc(C(C)(C)C)cc(C(C)(C)C)c1O</smiles>

7

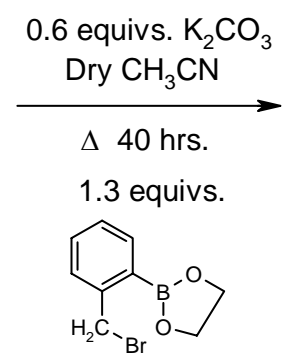

6<smiles>CC(C)(C)Cc1cc(C(C)(C)C)cc(C(C)(C)C)c1OCc1ccccc1B(O)O</smiles><smiles>[Z]C(C)(C)Cc1cc(C(C)(C)C)cc(Cc2cc(C(C)(C)C)cc(C(C)(C)C)c2OCc2ccccc2B(O)O)c1O</smiles>

Figure 2

The $p$-Hcalix[4]arene $\mathbf{1 0}$ was prepared from the corresponding $p$-tert-butylcalix[4]arene $\mathbf{7}$ by reverse Friedel-Crafts reaction with aluminium chloride employing the method of Gutsche and co-workers. $^{15,16}$

Using similar methodology to that described for the p-tert-butyl derivative 7, selective boronation to the $p$-Hcalix[4]arene 10 was accomplished as shown in Figure 3. Monoboronic acid $\mathbf{1 1}$ and diboronic acid $\mathbf{1 2}$ calixarenes were prepared in this manner. 
<smiles>CC(C)(C)Cc1cccc(C(C)(C)C)c1O</smiles>

10<smiles>CC(C)(C)Cc1cccc(C(C)(C)C)c1O</smiles>

10
0.6 equivs. $\mathrm{K}_{2} \mathrm{CO}_{3}$
Dry $\mathrm{CH}_{3} \mathrm{CN}$

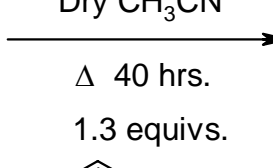<smiles>C1=COB2OCCOC2=C1</smiles>

6<smiles>COc1c(CC(C)(C)C)ccc(CC(C)(C)C)c1O</smiles><smiles>OCc1ccccc1B(O)O</smiles><smiles>[Z]C(C)(C)Cc1ccccc1COc1c(Cc2ccccc2B(O)O)cccc1C(C)(C)C</smiles>

\section{Figure 3}

Incorporation of the $p$-allyl functionality was achieved by treatment of $p$-Hcalix[4]arene 10 with allyl bromide affording the lower rim functionalized $o$-allyl derivative. Claisen rearrangement in the presence of $\mathrm{N}, \mathrm{N}$-diethylamine ${ }^{\mathbf{1 5 , 1 6}}$ furnished the desired $p$-allylcalix[4]arene $\mathbf{1 3}$ as shown in Figure 4.

Significant intramolecular H-bonding between the boronic acid moiety and the unfunctionalised phenolic $\mathrm{OH}$ groups was spectrally exhibited (by ${ }^{1} \mathrm{H}$ NMR) for each new ligand. Prolonged heating in water was required to disrupt this effect.

${ }^{1} \mathrm{H}$ NMR analysis was used to establish that each of the illustrated examples exist in a cone shaped conformation. 1,3-Distal difunctionalization is observed for each of the diboronic acids.

These first examples of lower rim functionalized boronic acid calixarenes with the upper rim functionalized with allyl rather than alkyl substituents were prepared to allow further study of guest complexation in non-aqueous, aqueous and on solid substrates. Allyl group derivatization at the upper rim can be used as a means for attachment of these calixarene analogues to solid supports. $^{17}$ 
<smiles>C=CCc1cc(CC(C)(C)C)c(O)c(C(C)(C)C)c1</smiles>

13

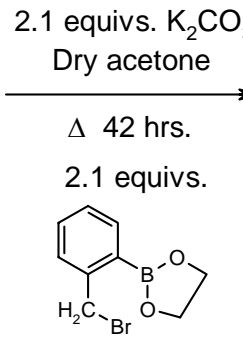

6<smiles>C=CCc1cc(CC=C)c(OCc2ccccc2B(O)O)c(CC(C)(C)C)c1</smiles><smiles>C=CCc1cc(Cc2cc(CC=C)cc(C(C)(C)C)c2OCc2ccccc2B(O)O)c(O)c(CC(C)(C)C)c1</smiles>

\section{Figure 4}

Solution state spectroscopic investigation of the complexing abilities of the calixarene boronic acid compounds indicated some degree of molecular recognition towards simple amines, aminoalcohols and diols. Complexation studies were extended to more complex diol systems and a very promising result was obtained with p-tert-butylcalix[4]arene diboronic acid, which was observed to complex D-glucose in $\mathrm{CDCl}_{3}$ by ${ }^{1} \mathrm{H}$-NMR spectroscopy. In particular, four new tertbutyl signals appear between 0.8 and $1.5 \mathrm{ppm}$. This spectral arrangement implies that the complexation of an asymmetric guest takes place on interaction with D-glucose, conferring different chemical environments to each monomer unit of the cyclic calixarene substructure.

Further synthesis and detailed spectroscopic investigations of guest complexation are needed however to fully understand the chemistry involved, including the effects of upper and lower rim functionalization on the nature and selectivity of guest complexation. It may then be possible to design boronic acid calixarenes to selectively recognise certain diol functionalities leading, ultimately, to very interesting and potentially useful macrocycles. 


\section{Experimental Section}

Tri-n-butyl borate (3). A mixture of boric acid (20.00 g, $0.32 \mathrm{~mol})$ and $n$-butanol (100 mL, $1.09 \mathrm{~mol}$ ) was heated and distilled with collection of the following fractions: $\mathrm{H}_{2} \mathrm{O}: n$-butanol azeotrope, b.p. $95{ }^{\circ} \mathrm{C} ; \mathrm{H}_{2} \mathrm{O}$, b.p. $100{ }^{\circ} \mathrm{C}$; $n$-butanol, b.p. $118{ }^{\circ} \mathrm{C}$; and finally, tri-n-butyl borate 3, b.p. $71{ }^{\circ} \mathrm{C} 20.5 \mathrm{~mm} \mathrm{Hg}$. The product was obtained as a colourless liquid in quantitative yield (70.15 g). [Found: $\mathrm{m} / \mathrm{z}(\mathrm{EI}): \mathrm{M}^{+}$230.2059. $\mathrm{C}_{12} \mathrm{H}_{27} \mathrm{O}_{3} \mathrm{~B}$ requires $\mathrm{M}^{+}$230.2053]; $\delta_{\mathrm{H}}\left(\mathrm{CDCl}_{3}\right)$, $(300 \mathrm{MHz}) 0.93\left(9 \mathrm{H}, \mathrm{q}, \mathrm{J}=4.9 \mathrm{~Hz}, \mathrm{CH}_{3}\right), 1.37\left(6 \mathrm{H}, \mathrm{m}, \mathrm{OCH}_{2} \mathrm{CH}_{2} \mathrm{CH}_{2} \mathrm{CH}_{3}\right), 1.51(6 \mathrm{H}, \mathrm{m}$, $\left.\mathrm{OCH}_{2} \mathrm{CH}_{2} \mathrm{CH}_{2} \mathrm{CH}_{3}\right), 3.77$ (6H, t, $\left.\mathrm{J}=6.5 \mathrm{~Hz}, \mathrm{OCH}_{2} \mathrm{CH}_{2} \mathrm{CH}_{2} \mathrm{CH}_{3}\right)$.

2-Methylphenyl boronic acid (4). To a stirring mixture of magnesium turnings (2.84 g, $0.12 \mathrm{~mol}$ ) in dry THF (40 mL) under dry $\mathrm{N}_{2}$ was added dropwise, over a period of 30 mins., a solution of $o$-bromotoluene $(20.00 \mathrm{~g}, 0.12 \mathrm{~mol})$ in dry THF $(20 \mathrm{~mL})$ from a pressure-equalizing dropping funnel. A crystal of iodine and a heat gun were used initially to directly heat the reaction flask, encouraging Grignard formation. Once the addition was complete, the reaction was heated under reflux for $2 \mathrm{hr}$, providing the required Grignard as a clear brown liquid. This Grignard and tri-n-butyl borate $3(26.86 \mathrm{~mL}, 0.12 \mathrm{~mol})$ were added dropwise simultaneously to dry THF $(100 \mathrm{~mL})$ in a 1 litre three-necked round bottomed flask with mechanical stirrer attached. The reaction mixture was maintained at $-70{ }^{\circ} \mathrm{C}$ (ethyl acetate:liquid $\mathrm{N}_{2}$ bath) for the duration of the addition. Once complete the reaction mixture was allowed to warm to room temperature, revealing a clear pale brown liquid. The product was hydrolysed by the addition of distilled water $(100 \mathrm{~mL})$ and vigorous stirring overnight. The resulting grey precipitate was filtered through a bed of Celite and the filtrate was concentrated to dryness under reduced pressure to furnish the desired boronic acid $\mathbf{4}$ as a white solid. Recrystallization from ice-cold water afforded analytically pure 4 as white flakes (8.91 g, 56\%). [Found: m/z (EI): $\mathrm{M}^{+}$354.0. $\mathrm{C}_{7} \mathrm{H}_{9} \mathrm{O}_{2} \mathrm{~B}$ requires $\mathrm{M}^{+}$135.9. $\left(\mathrm{C}_{7} \mathrm{H}_{7} \mathrm{OB}\right)_{3}$, anhydride, requires $\mathrm{M}^{+}$353.6]; vmax. $\left(\mathrm{KBr} / \mathrm{cm}^{-1}\right) 3455$ $(\mathrm{OH}) ; \delta_{\mathrm{H}}\left(\mathrm{CDCl}_{3}\right),(300 \mathrm{MHz}) 2.82\left(3 \mathrm{H}, \mathrm{s}, \mathrm{CH}_{3}\right), 7.29(2 \mathrm{H}, \mathrm{m}, \mathrm{ArH}), 7.45(1 \mathrm{H}, \mathrm{t}, \mathrm{J}=7.4 \mathrm{~Hz}$, $\mathrm{ArH}), 8.22(1 \mathrm{H}, \mathrm{d}, \mathrm{J}=7.4 \mathrm{~Hz}, \mathrm{ArH})$.

2-Methylphenylethyleneboronate (5). A mixture of 2-methylphenyl boronic acid 4 (3.50 g, $25.76 \mathrm{mmol})$ and ethylene glycol (1.60 g, $25.76 \mathrm{mmol})$ in dry benzene $(50 \mathrm{~mL})$ was refluxed for 2 hrs. using a Dean-Stark apparatus for water removal. At this stage approx. $1 \mathrm{~mL}$ of water was recovered. The reaction mixture was concentrated at reduced pressure to yield $\mathbf{5}$ as a colourless liquid in quantitative yield (4.10 g). [Found: $\mathrm{C}, 66.8 ; \mathrm{H}, 7.0 . \mathrm{C}_{9} \mathrm{H}_{11} \mathrm{O}_{2} \mathrm{~B}$ requires $\mathrm{C}, 66.7, \mathrm{H}$, 6.8\%]; [Found: $\mathrm{m} / \mathrm{z}$ (EI): $\mathrm{M}^{+}$162.0852. $\mathrm{C}_{9} \mathrm{H}_{11} \mathrm{O}_{2} \mathrm{~B}$ requires $\mathrm{M}^{+}$162.0856]; $\delta_{\mathrm{H}}\left(\mathrm{CDCl}_{3}\right)$, (300MHz) $2.55\left(3 \mathrm{H}, \mathrm{s}, \mathrm{CH}_{3}\right), 4.37\left(4 \mathrm{H}, \mathrm{s}, \mathrm{OCH}_{2}\right), 7.19(2 \mathrm{H}, \mathrm{m}, \mathrm{ArH}), 7.34(1 \mathrm{H}, \mathrm{t}, \mathrm{J}=7.5 \mathrm{~Hz}$, $\operatorname{ArH}), 7.80(1 \mathrm{H}, \mathrm{d}, \mathrm{J}=7.4 \mathrm{~Hz}$, ArH).

2-Bromomethylphenylethyleneboronate (6). A mixture of 2-methylphenylethyleneboronate 5 (4.10 g, $25.30 \mathrm{mmol}$ ), N-bromosuccinamide (NBS) (4.50 g, $25.30 \mathrm{mmol}$ ) and a catalytic amount of AIBN in carbontetrachloride $(75 \mathrm{~mL})$ was heated with stirring. Upon reaching the reflux temperature bromine evolution was observed. After $5 \mathrm{~min}$. bromine evolution was no longer visible. The reaction mixture was a pale orange colour at this stage. After refluxing for 2 hrs., the 
reaction mixture was cooled to room temperature and subsequently cooled to $0{ }^{\circ} \mathrm{C}$ with an ice bath. The precipitated succinimide was removed by filtration and the filtrate concentrated under reduced pressure to furnish $\mathbf{6}$ as a yellow oil which crystallized to a pale yellow solid (4.86 g, $80 \%$ ) upon cooling to $-15{ }^{\circ} \mathrm{C}$. A melting point was not recorded because the product sublimes. [Found: $\mathrm{C}, 44.4 ; \mathrm{H}, 3.9$. $\mathrm{C}_{9} \mathrm{H}_{10} \mathrm{O}_{2} \mathrm{BBr}$ requires $\mathrm{C}, 44.9 ; \mathrm{H}, 4.2 \%$ ]; [Found: $\mathrm{m} / \mathrm{z}$ (EI): $\mathrm{M}^{+}$ 239.9957. $\mathrm{C}_{9} \mathrm{H}_{10} \mathrm{O}_{2} \mathrm{BBr}$ requires $\left.\mathrm{M}^{+} 239.9948\right] ; \delta_{\mathrm{H}}\left(\mathrm{CDCl}_{3}\right),(300 \mathrm{MHz}) 4.43\left(4 \mathrm{H}, \mathrm{s}, \mathrm{OCH}_{2}\right), 4.91$ (2H, s, $\left.\mathrm{CH}_{2} \mathrm{Br}\right), 7.32(1 \mathrm{H}, \mathrm{t}, \mathrm{J}=7.4 \mathrm{~Hz}, \mathrm{ArH}), 7.44(2 \mathrm{H}, \mathrm{m}, \mathrm{ArH}), 7.86(1 \mathrm{H}, \mathrm{d}, \mathrm{J}=7.3 \mathrm{~Hz}, \operatorname{ArH})$.

p-tert-Butylcalix[4]arene monoboronic acid (8). A mixture of p-tert-butylcalix[4]arene 7 (2.50 g, $3.86 \mathrm{mmol})$, 2-bromo-methylphenylethyleneboronate 6 (1.23 g, $5.10 \mathrm{mmol})$ and potassium carbonate $(0.32 \mathrm{~g}, 2.31 \mathrm{mmol})$ in dry acetonitrile $(100 \mathrm{~mL})$ were heated under reflux in dry $\mathrm{N}_{2}$ conditions for 40 hrs. The pale yellow-coloured solution was allowed to cool to room temperature and filtered through a bed of Celite. The residual inorganic salts were washed with methylene chloride and the filtrate concentrated to dryness. Methylene chloride ( $80 \mathrm{~mL}$ ) was added and the solution washed, in turn, with $1 \mathrm{~N} \mathrm{HCl}(2 \times 50 \mathrm{~mL})$ and distilled water $(1 \times 40 \mathrm{~mL})$. The organic layer was dried over $\mathrm{MgSO}_{4}$ and concentrated under reduced pressure to give an offwhite solid. The crude product was dissolved in ethyl acetate $(40 \mathrm{~mL})$ and after $c a$. 30 mins. a white powder (unreacted tetramer 7) ca. $0.5 \mathrm{~g}$ fell out of solution and was removed by filtration. The filtrate was concentrated to dryness and purified by flash column chromatography on silica gel, with methylene chloride:ethyl acetate (10:1) as eluent, to furnish monoboronic acid $\mathbf{8} \mathrm{R}_{\mathrm{f}} 0.71$ as a white solid (1.21 g, 40\%), m.p. 191-193 ${ }^{\circ} \mathrm{C}$. [Found: C, 76.5; H, 7.9. $\mathrm{C}_{51} \mathrm{H}_{63} \mathrm{O}_{6} \mathrm{~B} \cdot \mathrm{H}_{2} \mathrm{O}$ requires C, 76.5; $\mathrm{H}, 8.2 \%$ ]; vmax. $\left(\mathrm{KBr} / \mathrm{cm}^{-1}\right) 3435(\mathrm{OH})$; [Found: $\mathrm{m} / \mathrm{z}(\mathrm{ES}):(\mathrm{M}+\mathrm{H})^{+} 783.5$. $\mathrm{C}_{51} \mathrm{H}_{63} \mathrm{O}_{6} \mathrm{~B}$ requires $\mathrm{M}^{+} 782.3$ ]; $\delta_{\mathrm{H}}\left(\mathrm{CDCl}_{3}\right)$, $(300 \mathrm{MHz}) 1.20\left(36 \mathrm{H}, \mathrm{s}, \mathrm{C}\left(\mathrm{CH}_{3}\right)_{3}\right), 3.32\left(2 \mathrm{H}, \mathrm{d}, \mathrm{H}_{\mathrm{B}}\right.$, $\left.\mathrm{J}_{\mathrm{BA}}=13.2 \mathrm{~Hz}, \mathrm{ArCH}_{2} \mathrm{Ar}\right), 3.40\left(2 \mathrm{H}, \mathrm{d}, \mathrm{H}_{\mathrm{B}}, \mathrm{J}_{\mathrm{B}^{\prime} \mathrm{A}^{\prime}}=13.7 \mathrm{~Hz}, \operatorname{ArCH}_{2} \mathrm{Ar}\right), 4.18\left(4 \mathrm{H}, \mathrm{d}, \mathrm{H}_{\mathrm{A}}, \mathrm{H}_{\mathrm{A}^{\prime}}\right.$ overlapping, $\mathrm{J}=13.5 \mathrm{~Hz}, \operatorname{ArCH} \mathrm{Ar}), 5.18\left(2 \mathrm{H}, \mathrm{s}, \mathrm{OCH}_{2}\right), 6.97(2 \mathrm{H}, \mathrm{d}, \mathrm{J}=2.0 \mathrm{~Hz}, \mathrm{ArH}), 7.02(2 \mathrm{H}$, s, $\operatorname{ArH}), 7.05(2 \mathrm{H}, \mathrm{d}, \mathrm{J}=2.1 \mathrm{~Hz}, \operatorname{ArH}), 7.08(2 \mathrm{H}, \mathrm{s}, \operatorname{ArH}), 7.35(1 \mathrm{H}, \mathrm{m}, \operatorname{ArH}), 7.40(1 \mathrm{H}, \mathrm{t}, \mathrm{J}=$ 7.8Hz, $\operatorname{ArH}), 7.44(1 \mathrm{H}, \mathrm{t}, \mathrm{J}=7.4 \mathrm{~Hz}, \operatorname{ArH}), 7.79(1 \mathrm{H}, \mathrm{d}, \mathrm{J}=7.3 \mathrm{~Hz}, \operatorname{ArH}), 7.85(2 \mathrm{H}, \mathrm{s}, \mathrm{BOH})$, $9.54(2 \mathrm{H}, \mathrm{s}, \mathrm{OH}), 10.02(1 \mathrm{H}, \mathrm{s}, \mathrm{OH}) ; \delta_{\mathrm{B}}\left(\mathrm{CDCl}_{3} / \mathrm{BF}_{3} . \mathrm{Et}_{2} \mathrm{O}\right) 30.05$.

p-tert-Butylcalix[4]arene diboronic acid (9). A mixture of p-tert-butylcalix[4]arene 7 (1.00 g, $1.54 \mathrm{mmol})$, 2-bromo-methylphenylethyleneboronate $6(0.78 \mathrm{~g}, 3.24 \mathrm{mmol})$ and potassium carbonate $(0.45 \mathrm{~g}, 3.24 \mathrm{mmol})$ in dry acetone $(50 \mathrm{~mL})$ were heated under reflux in dry $\mathrm{N}_{2}$ conditions for 24 hrs. The reaction mixture was worked up as described for $\mathbf{8}$ affording the crude diboronic acid as an off-white solid. Purification by flash column chromatography on silica gel, eluting with methylene chloride:ethyl acetate (10:1), furnished analytically pure $\mathbf{9} \mathrm{R}_{\mathrm{f}} 0.27$ as a white solid (0.48 g, 34\%), m.p. $242-244{ }^{\circ} \mathrm{C}$ and additionally, the monoboronated derivative $8 \mathrm{R}_{\mathrm{f}}$ 0.71 (0.33 g, 27\%). [Found: C, 75.9; $\mathrm{H}, 7.5 . \mathrm{C}_{58} \mathrm{H}_{70} \mathrm{O}_{8} \mathrm{~B}_{2}$ requires $\mathrm{C}, 76.0 ; \mathrm{H}, 7.7 \%$ ]; vmax. $\left(\mathrm{KBr} / \mathrm{cm}^{-1}\right) 3434(\mathrm{OH})$; [Found: $\mathrm{m} / \mathrm{z}(\mathrm{ES}):\left(\mathrm{M}+\mathrm{H}_{2} \mathrm{O}\right)^{+}$933.8, $(\mathrm{M}+\mathrm{Na})^{+}$939.2, $(\mathrm{M}+\mathrm{K})^{+}$955.4. $\mathrm{C}_{58} \mathrm{H}_{70} \mathrm{O}_{8} \mathrm{~B}_{2}$ requires $\mathrm{M}^{+}$916.1]; $\delta_{\mathrm{H}}\left(\mathrm{CDCl}_{3}\right),(300 \mathrm{MHz}) 0.94\left(18 \mathrm{H}, \mathrm{s}, \mathrm{C}\left(\mathrm{CH}_{3}\right)_{3}\right), 1.24(18 \mathrm{H}, \mathrm{s}$, $\left.\mathrm{C}\left(\mathrm{CH}_{3}\right)_{3}\right), 2.95\left(4 \mathrm{H}, \mathrm{d}, \mathrm{H}_{\mathrm{B}}, \mathrm{J}_{\mathrm{BA}}=13.3 \mathrm{~Hz}, \mathrm{ArCH}_{2} \mathrm{Ar}\right), 3.75\left(4 \mathrm{H}, \mathrm{d}, \mathrm{H}_{\mathrm{A}}, \mathrm{J}_{\mathrm{AB}}=13.3 \mathrm{~Hz}, \mathrm{ArCH} \mathrm{H}_{2} \mathrm{Ar}\right)$, 5.29 (4H, bs, $\left.\mathrm{OCH}_{2}\right), 6.70(2 \mathrm{H}, \mathrm{d}, \mathrm{J}=7.8 \mathrm{~Hz}, \operatorname{ArH}), 6.75(4 \mathrm{H}, \mathrm{s}, \operatorname{ArH}), 6.95(4 \mathrm{H}, \mathrm{s}, \mathrm{ArH}), 7.21$ 
$(2 \mathrm{H}, \mathrm{t}, \mathrm{J}=7.3 \mathrm{~Hz}, \operatorname{ArH}), 7.36(2 \mathrm{H}, \mathrm{t}, \mathrm{J}=7.5 \mathrm{~Hz}, \mathrm{ArH}), 7.74(2 \mathrm{H}, \mathrm{s}, \mathrm{OH}), 7.83(2 \mathrm{H}, \mathrm{d}, \mathrm{J}=7.5 \mathrm{~Hz}$, $\mathrm{ArH}), 8.19$ (4H, bs, $\mathrm{BOH}) ; \delta_{\mathrm{B}}\left(\mathrm{CDCl}_{3} / \mathrm{BF}_{3} . \mathrm{Et}_{2} \mathrm{O}\right) 31.53$.

p-Hcalix[4]arene monoboronic acid (11). A mixture of p-Hcalix[4]arene 10 (1.50 g, $3.54 \mathrm{mmol})$, 2-bromomethyl-phenylethyleneboronate 6 (1.10 g, $4.60 \mathrm{mmol})$ and potassium carbonate $(0.29 \mathrm{~g}, 2.12 \mathrm{mmol})$ in dry acetonitrile $(60 \mathrm{~mL})$ were heated under reflux in dry $\mathrm{N}_{2}$ conditions for $40 \mathrm{hrs}$. The reaction mixture was worked up as described for $\mathbf{8}$ affording the crude monoboronic acid as an off-white solid. Purification by flash column chromatography on silica gel, eluting with methylene chloride:ethyl acetate (10:1), furnished analytically pure $11 \mathrm{R}_{\mathrm{f}} 0.85$ as a white solid (0.87 g, 44\%), m.p. 200-202 ${ }^{\circ} \mathrm{C}$. [Found: C, 75.6; H, 5.6. $\mathrm{C}_{35} \mathrm{H}_{31} \mathrm{O}_{6} \mathrm{~B}$ requires $\mathrm{C}$, 75.3; H, 5.6\%]; vmax. (KBr/cm $\left.{ }^{-1}\right) 3417(\mathrm{OH})$; [Found: $\mathrm{m} / \mathrm{z}(\mathrm{ES}): \mathrm{M}^{+}$558.6, $(\mathrm{M}+\mathrm{H})^{+}$559.6, $\left(\mathrm{M}+\mathrm{H}_{2} \mathrm{O}\right)^{+}$575.9, $(\mathrm{M}+\mathrm{Na})^{+}$581.3, $(\mathrm{M}+\mathrm{K})^{+}$597.3. $\mathrm{C}_{35} \mathrm{H}_{31} \mathrm{O}_{6} \mathrm{~B}$ requires $\mathrm{M}^{+}$558.2]; $\delta_{\mathrm{H}}\left(\mathrm{CDCl}_{3}\right)$, $(300 \mathrm{MHz}) 3.29\left(2 \mathrm{H}, \mathrm{d}, \mathrm{H}_{\mathrm{B}^{\prime}}, \mathrm{J}_{\mathrm{B}^{\prime} \mathrm{A}^{\prime}}=13.4 \mathrm{~Hz}, \operatorname{ArCH} \mathrm{H}_{2} \mathrm{Ar}\right) 3.44\left(2 \mathrm{H}, \mathrm{d}, \mathrm{H}_{\mathrm{B}}, \mathrm{J}_{\mathrm{BA}}=13.9 \mathrm{~Hz}\right.$, $\left.\mathrm{ArCH}_{2} \mathrm{Ar}\right), 4.08\left(2 \mathrm{H}, \mathrm{d}, \mathrm{H}_{\mathrm{A}^{\prime}}, \mathrm{J}_{\mathrm{A}^{\prime} \mathrm{B}^{\prime}}=13.3 \mathrm{~Hz}, \operatorname{ArCH} \mathrm{Ar}\right), 4.20\left(2 \mathrm{H}, \mathrm{d}, \mathrm{H}_{\mathrm{A}}, \mathrm{J}_{\mathrm{AB}}=13.9 \mathrm{~Hz}\right.$, $\left.\mathrm{ArCH}_{2} \mathrm{Ar}\right), 5.25$ (2H, s, OCH $\mathrm{OCH}_{2}, 6.23(2 \mathrm{H}, \mathrm{s}, \mathrm{BOH}), 6.68(3 \mathrm{H}, \mathrm{m}, \mathrm{ArH}), 6.89(1 \mathrm{H}, \mathrm{t}, \mathrm{J}=7.3 \mathrm{~Hz}$, $\operatorname{ArH}), 6.96$ (2H, d, J = 7.5Hz, ArH), 7.03 (6H, m, ArH), $7.17(1 \mathrm{H}, \mathrm{d}, \mathrm{J}=7.4 \mathrm{~Hz}, \mathrm{ArH}), 7.42(1 \mathrm{H}$, $\mathrm{t}, \mathrm{J}=7.5 \mathrm{~Hz}, \operatorname{ArH}), 7.47(1 \mathrm{H}, \mathrm{t}, \mathrm{J}=7.3 \mathrm{~Hz}, \operatorname{ArH}), 7.84(1 \mathrm{H}, \mathrm{d}, \mathrm{J}=7.3 \mathrm{~Hz}, \operatorname{ArH}), 9.22(2 \mathrm{H}, \mathrm{s}, \mathrm{OH})$, $9.43(1 \mathrm{H}, \mathrm{s}, \mathrm{OH}) ; \delta_{\mathrm{B}}\left(\mathrm{CDCl}_{3} / \mathrm{BF}_{3} . \mathrm{Et}_{2} \mathrm{O}\right) 29.91$.

p-Hcalix[4]arene diboronic acid (12). A mixture of p-Hcalix[4]arene 10 (1.50 g, $3.54 \mathrm{mmol})$, 2-bromomethyl-phenylethyleneboronate 6 (1.79 g, $7.43 \mathrm{mmol}$ ) and potassium carbonate (1.02 g, $7.43 \mathrm{mmol})$ in dry acetone $(60 \mathrm{~mL})$ were heated under reflux in dry $\mathrm{N}_{2}$ conditions for $24 \mathrm{hrs}$. The reaction mixture was worked up as described for $\mathbf{8}$ affording the crude diboronic acid as an offwhite solid. Purification by flash column chromatography on silica gel, eluting with methylene chloride:ethyl acetate (10:1), furnished analytically pure $12 \mathrm{R}_{\mathrm{f}} 0.38$ as a white solid (1.05 g, 43\%), m.p. 262-264 ${ }^{\circ} \mathrm{C}$ and additionally, the monoboronated derivative $11 \mathrm{R}_{\mathrm{f}} 0.85$ (0.65 g, 33\%). [Found: C, 70.9; H, 5.4. $\mathrm{C}_{42} \mathrm{H}_{38} \mathrm{O}_{8} \mathrm{~B}_{2} \cdot \mathrm{H}_{2} \mathrm{O}$ requires $\mathrm{C}$, 71.0; $\mathrm{H}, 5.7 \%$ ]; vmax. $\left(\mathrm{KBr} / \mathrm{cm}^{-1}\right) 3428$ $(\mathrm{OH})$; [Found: $\mathrm{m} / \mathrm{z}(\mathrm{ES}):(\mathrm{M}+\mathrm{Na})^{+}$714.7, $(\mathrm{M}+\mathrm{K})^{+}$731.0. $\mathrm{C}_{42} \mathrm{H}_{38} \mathrm{O}_{8} \mathrm{~B}_{2}$ requires $\mathrm{M}^{+}$691.9]; $\delta_{\mathrm{H}}$ $\left(\mathrm{CDCl}_{3}\right),(300 \mathrm{MHz}) 3.02\left(4 \mathrm{H}, \mathrm{d}, \mathrm{H}_{\mathrm{B}}, \mathrm{J}_{\mathrm{BA}}=13.1 \mathrm{~Hz}, \mathrm{ArCH}_{2} \mathrm{Ar}\right), 3.78\left(4 \mathrm{H}, \mathrm{d}, \mathrm{H}_{\mathrm{A}}, \mathrm{J}_{\mathrm{AB}}=13.3 \mathrm{~Hz}\right.$, $\left.\mathrm{ArCH}_{2} \mathrm{Ar}\right), 5.36$ (4H, bs, $\left.\mathrm{OCH}_{2}\right), 6.64(2 \mathrm{H}, \mathrm{t}, \mathrm{J}=7.9 \mathrm{~Hz}, \mathrm{ArH}), 6.65(2 \mathrm{H}, \mathrm{d}, \mathrm{J}=7.7 \mathrm{~Hz}, \operatorname{ArH}), 6.75$ $(2 \mathrm{H}, \mathrm{t}, \mathrm{J}=7.4 \mathrm{~Hz}, \operatorname{ArH}), 6.86(4 \mathrm{H}, \mathrm{d}, \mathrm{J}=7.3 \mathrm{~Hz}, \operatorname{ArH}), 6.97(4 \mathrm{H}, \mathrm{d}, \mathrm{J}=7.5 \mathrm{~Hz}, \operatorname{ArH}), 7.22(2 \mathrm{H}, \mathrm{t}$, $\mathrm{J}=7.5 \mathrm{~Hz}, \operatorname{ArH}), 7.38(2 \mathrm{H}, \mathrm{t}, \mathrm{J}=7.3 \mathrm{~Hz}, \operatorname{ArH}), 7.85(2 \mathrm{H}, \mathrm{d}, \mathrm{J}=6.7 \mathrm{~Hz}, \operatorname{ArH}), 8.20(2 \mathrm{H}, \mathrm{s}, \mathrm{OH})$, $8.24(4 \mathrm{H}, \mathrm{bs}, \mathrm{BOH}) ; \delta_{\mathrm{B}}\left(\mathrm{CDCl}_{3} / \mathrm{BF}_{3} . \mathrm{Et}_{2} \mathrm{O}\right) 31.70$.

\section{p-Allyl-calix[4]arene monoboronic acid (14) and p-allyl-calix[4]arene diboronic acid (15). A} mixture of $p$-allyl-calix[4]arene 13 (2.88 g, $4.93 \mathrm{mmol}$ ), 2-bromomethyl-phenylethyleneboronate 6 (2.48 g, $10.40 \mathrm{mmol})$ and potassium carbonate $(1.43 \mathrm{~g}, 10.40 \mathrm{mmol})$ in dry acetone $(150 \mathrm{~mL})$ were heated under reflux in dry $\mathrm{N}_{2}$ conditions for $42 \mathrm{hrs}$. The reaction mixture was worked up as described for $\mathbf{8}$ affording the crude calixboronic acids as an off-white solid. Purification by flash column chromatography on silica gel, eluting with methylene chloride:ethyl acetate (10:1), furnished the monoboronated derivative $14 \mathrm{R}_{\mathrm{f}} 0.17$ as an off-white solid (0.66 g, 19\%), and additionally, the diboronated derivative $15 \mathrm{R}_{\mathrm{f}} 0.81$ (0.88 g, 21\%). Both isolated solids were heated in water for 2 hours to disrupt intramolecular H-bonding which ultimately affects 
subsequent NMR analysis. Compound 14: $\delta_{\mathrm{H}}\left(\mathrm{CDCl}_{3}\right),(270 \mathrm{MHz}) 3.16\left(8 \mathrm{H}, \mathrm{m}, \mathrm{CH}_{2}-\mathrm{CH}=\mathrm{CH}_{2}\right)$, $3.24\left(2 \mathrm{H}, \mathrm{d}, \mathrm{H}_{\mathrm{B}}{ }^{\prime}, \mathrm{J}_{\mathrm{B}}{ }^{\prime} \mathrm{A}^{\prime}=14.3 \mathrm{~Hz}, \mathrm{ArCH} \mathrm{Ar}_{2} \mathrm{Ar}, 3.36\left(2 \mathrm{H}, \mathrm{d}, \mathrm{H}_{\mathrm{B}}, \mathrm{J}_{\mathrm{BA}}=16.9 \mathrm{~Hz}, \mathrm{ArCH} \mathrm{Ar}_{2}\right), 4.06(2 \mathrm{H}\right.$, d, $\left.\mathrm{H}_{\mathrm{A}^{\prime}}, \mathrm{J}_{\mathrm{A}^{\prime} \mathrm{B}}{ }^{\prime}=12.7 \mathrm{~Hz}, \mathrm{ArCH} \mathrm{Ar}\right), 4.14\left(2 \mathrm{H}, \mathrm{d}, \mathrm{H}_{\mathrm{A}}, \mathrm{J}_{\mathrm{AB}}=16.9 \mathrm{~Hz}, \mathrm{ArCH} \mathrm{H}_{2} \mathrm{Ar}\right), 5.05\left(8 \mathrm{H}, \mathrm{m}, \mathrm{CH}_{2^{-}}\right.$ $\left.\mathrm{CH}=\mathrm{CH}_{2}\right), 5.20\left(2 \mathrm{H}, \mathrm{s}, \mathrm{OCH}_{2}\right), 5.84\left(4 \mathrm{H}, \mathrm{m}, \mathrm{CH}_{2}-\mathrm{CH}=\mathrm{CH}_{2}\right), 6.33(2 \mathrm{H}, \mathrm{s}, \mathrm{BOH}), 6.78(2 \mathrm{H}, \mathrm{s}$, ArH calix), 6.79 (2H, d, J = 2.5Hz, ArH calix), 6.82 (2H, d, J = 1.5Hz, ArH calix), 6.84 (2H, s, ArH calix), $7.21(1 \mathrm{H}, \mathrm{d}, \mathrm{J}=7.6 \mathrm{~Hz}, \operatorname{ArH}), 7.45(2 \mathrm{H}, \mathrm{m}, \operatorname{ArH}), 7.81(1 \mathrm{H}, \mathrm{d}, \mathrm{J}=7.4 \mathrm{~Hz}, \operatorname{ArH}), 9.28$ $(2 \mathrm{H}, \mathrm{s}, \mathrm{OH}), 9.57(1 \mathrm{H}, \mathrm{s}, \mathrm{OH})$. Compound 15: $\delta_{\mathrm{H}}\left(\mathrm{CDCl}_{3}\right),(270 \mathrm{MHz}) 2.98\left(4 \mathrm{H}, \mathrm{d}, \mathrm{H}_{\mathrm{B}}\right.$, $\left.\mathrm{ArCH}_{2} \mathrm{Ar}\right), 3.08\left(4 \mathrm{H}, \mathrm{d}, \mathrm{CH}_{2}-\mathrm{CH}=\mathrm{CH}_{2}\right), 3.21\left(4 \mathrm{H}, \mathrm{d}, \mathrm{CH}_{2}-\mathrm{CH}=\mathrm{CH}_{2}\right), 3.72\left(2 \mathrm{H}, \mathrm{d}, \mathrm{H}_{\mathrm{A}}\right.$, $\left.\mathrm{ArCH}_{2} \mathrm{Ar}\right), 4.86-5.02\left(8 \mathrm{H}, \mathrm{m}, \mathrm{CH}_{2}-\mathrm{CH}=\mathrm{CH}_{2}\right), 5.30\left(4 \mathrm{H}, \mathrm{bs}, \mathrm{OCH}_{2}\right), 5.79-5.86\left(4 \mathrm{H}, \mathrm{m}, \mathrm{CH}_{2}-\right.$ $\mathrm{CH}=\mathrm{CH}_{2}$ ), 6.65 (4H, s, ArH calix), 6.71 (4H, s, ArH calix), 6.78 (2H, s, OH), 7.23 (2H, t, ArH), 7.35 (4H, m, ArH), 7.82 (2H, d, ArH), 8.11 (4H, s, BOH).

\section{Acknowledgements}

Research grant support from Enterprise Ireland through Basic and Strategic Research Programs is gratefully acknowledged.

\section{References}

1. Czarnik, A.W. Acc. Chem. Res. 1994, 27, 302.

2. Pizer, R. Polyhedron 1996, 15, 3411.

3. Hagemeier, E.; Kemper, K; Boos, K.-S.; Schlimme, E. J. Chromat. 1983, 282, 663.

4. Toi, H.; Nagai, Y.; Aoyama, Y.; Kawabe, H.; Aizawa, K.; Ogoshi, H. Chem. Lett. 1993, 1040.

5. Schwing-Weill, M.J.; McKervey, M.A. In Calixarenes: A Versatile Class of Macrocyclic Compounds, Vincens, J.; Bohmer, V. Eds; Kluwer Academic Publishers: Dordrecht, 1991.

6. Brindle, R.; Albert, K.; Harris, S.J.; Troltzsch, C.; Horne, E.; Glennon, J.D. Silica Bonded Calixarenes In Chromatography-I. Synthesis and Characterisation by Solid State NMR Spectroscopy J. Chromatography A 1996, 731, 41.

7. Glennon, J.D.; Horne, E.; Hall, K.; Cocker, D.; Kuhn, A.; Harris, S.J.; McKervey, M.A. Silica Bonded Calixarenes in Chromatography-II. Chromatographic Retention of Metal Ions and Amino Acid Ester Hydrochlorides J. Chromatography A 1996, 731, 47.

8. (a) Glennon, J.D.; Hutchinson, S.; McSweeney, C.C.; Harris, S.J.; McKervey, M.A. Molecular Baskets in Supercritical $\mathrm{CO}_{2}$ Analytical Chemistry 69(11) (1997) 2207. (b) Glennon, J.D.; Harris, S.J.; Walker, A.; McSweeney, C.C.; O'Connell, M. Carrying Gold in Supercritical $\mathrm{CO}_{2}$ Gold Bulletin 1999, 32, 52. 
9. Glennon, J.D. Molecular Recognition Technology - Inorganic Extraction In Encyclopedia of Separation Science, Academic Press, Wilson, I.A.; Adlard, T.R.; Poole, C.F.; Cook, M., Eds; III 2000, 3400.

10. Linnane, P.; James, T.D.; Shinkai, S. J. Chem. Soc., Chem. Commun. 1995, 1997.

11. Tuladhar. S.M.; D’Silva, C. Tetrahedron Lett. 1992, 33, 265.

12. Tompkins, J. Org. Synth. 1933,13,16.

13. Eggers, C.A.; Kettle, S.F.A. Inorg. Chem. 1967, 6, 1975.

14. Groenen, L.C.; Ruel, B.H.M.; Casnati, A.; Verboom, W.; Pochini, A.; Ungaro, R.; Reinhoudt, D.N. Tetrahedron 1991, 47, 8379.

15. Gutsche, C.D.; Levine, J.A. J. Am. Chem. Soc. 1982, 104, 2652.

16. Gutsche, C.D.; Levine, J.A.; Sujeeth, P.K. J. Org. Chem. 1985, 50, 5802.

17. Hutchinson, S.; Kearney, G.A.; Horne, E.; Lynch, B.; Glennon, J.D.; McKervey, M.A.; Harris, S.J. Anal. Chim. Acta. 1994, 291, 269. 